\title{
Pulmonary alveolar microlithiasis: A case report and review of the literature
}

\author{
XU-DONG ZHANG ${ }^{1}$, JIN-MING GAO ${ }^{1}$, JIN-MEI LUO ${ }^{1}$ and YU ZHAO $^{2}$ \\ Departments of ${ }^{1}$ Respiratory Disease and ${ }^{2}$ Pathology, Peking Union Medical College Hospital, Beijing 100730, P.R. China
}

Received March 25, 2017; Accepted August 1, 2017

DOI: $10.3892 /$ etm.2017.5457

\begin{abstract}
Pulmonary alveolar microlithiasis (PAM) is a rare autosomal recessive disease characterized by the formation of calcium phosphate microliths in the alveoli. Mutations in the gene encoding the solute carrier family 34 member 2 gene are considered to be involved in the pathogenesis of PAM. Although PAM can develop in children, the majority of patients with PAM are diagnosed in adulthood due to the slow progressive nature of the disease within the lungs. In childhood, the majority of patients with PAM are asymptomatic and changes in the lung parenchyma are usually identified incidentally. Symptoms of PAM typically appear in the third or fourth decade of life and there is often a notable dissociation between the advanced radiological findings and the mild clinical presentation. A positive diagnosis of PAM is reached by the combination of a positive chest radiograph and histological examination. Genetic testing may help to identify other latent patients in the family of the patient with PAM. In the present study, the cases of 3 patients diagnosed with PAM have been reported, including their clinical presentation, radio imaging, pathological symptoms, genetic test results and treatment plans, as well as the associated literature.
\end{abstract}

\section{Introduction}

Pulmonary alveolar microlithiasis (PAM) is a hereditary lung disease in which calcium phosphate microliths, termed calcospherites, accumulate in the alveolar spaces (1). Mutations in the solute carrier family 34 member 2 (SLC34A2) gene, which encodes the type IIb sodium-phosphate cotransporter in alveolar type II cells, are responsible for the pathogenesis of PAM (2,3). SLC34A2 serves a crucial role in the transportation of phosphate ions from the alveolar spaces into alveolar

Correspondence to: Dr Jin-Ming Gao, Department of Respiratory Disease, Peking Union Medical College Hospital, 1 Shuaifuyuan Road, Beijing 100730, P.R. China

E-mail: gaojim@pumch.cn

Key words: pulmonary alveolar microlithiasis, clinical manifestations, diagnostic imaging, solute carrier family 34 member 2 type II cells (4). Mutations in the SLC34A2 gene that cause the dysfunction of alveolar type II cells result in the accumulation of phosphate and the formation of microliths in the alveolar spaces $(1,4)$. The majority of patients with PAM are asymptomatic at the time of diagnosis and the disease is usually identified incidentally following radiographic examination of the chest for other purposes (2). The typical presentation of PAM on a chest X-ray is by a 'sandstorm' appearance. In contrast to the radiological findings, which are often severe, the clinical presentation of PAM is often relatively mild or absent (5). In symptomatic patients shortness of breath is the most common symptom, followed by a dry cough, chest pain, sporadic hemoptysis and asthenia (1). Given the remarkable clinico-radiological dissociation, a positive diagnosis should be considered in a patient presenting with the typical radiological features of the disease without clinical symptoms, particularly in the presence of a family history or consanguinity (6). To the best of our knowledge, there is currently no definitive treatment available to prevent the progression of PAM, although lung transplantation has been used to treat patients with end-stage PAM (7) and disodium etidronate has been administered due to its alleged calcium phosphate precipitation-reducing effect in PAM; however, its use is controversial and its effectiveness is disputed $(8,9)$.

In the present study three cases of confirmed PAM are reported. All patients provided written informed consent. The epidemiology, cause, clinical features, radiological appearance, pathology, genetic mutations and treatment strategies for PAM were all considered.

\section{Case report}

Case 1. A 13-year-old female whose parents are first cousins (sharing 1/8 of their genes) presented to Peking Union Medical College Hospital, Beijing, China (PUMCH) in April 2016. Diffuse scattered nodules had been detected on a chest X-ray taken 4 years previously at her local hospital, however she was not diagnosed with PAM at that time. At the time of presentation the patient's physical examination was normal and they had no clinical complaints except exertional dyspnea. A chest X-ray revealed a bilaterally diffuse, fine sand-like micronodular appearance predominantly in the lower zones; high resolution computed tomography (HRCT) revealed widespread micro-calcifications throughout the lungs and pericardium with ground-glass opacities, interlobular septal 
thickening and pleural calcification (Fig. 1A and B). Broncho alveolar lavage fluid (BALF) was performed by squirting three $50 \mathrm{ml}$ samples of sterile saline into the right middle lobe of the lung and then immediately collecting it for testing. The sample was subsequently centrifuged at $250 \mathrm{xg}$ for $5 \mathrm{~min}$ at $4^{\circ} \mathrm{C}$. The supernatant was discarded and the sediments were prepared into smears and then fixed in 95\% alcohol (Beijing Jiu Zhou Bai Lin Biological Technology Co., Ltd., Beijing, China) at room temperature $\left(20-25^{\circ} \mathrm{C}\right)$ for $10 \mathrm{~min}$. The smears were stained with hematoxylin for $1 \mathrm{~min}$ and eosin for $20 \mathrm{sec}$ at room temperature $\left(20-25^{\circ} \mathrm{C}\right)$. Images were captured under an Eclipse 80i microscope (magnification, x400; Nikon Corporation, Tokyo, Japan). The quantities and classifications of inflammatory cells in the BALF sample were normal, but a concentrically laminated calcified body was identified (data not shown). Lung biopsy specimens were fixed in $10 \%$ neutral-buffered formalin at room temperature $\left(20-25^{\circ} \mathrm{C}\right.$; Beijing Jiu Zhou Bai Lin Biological Technology Co., Ltd.) overnight, cut into slices and dehydrated through a graded alcohol series, cleaned with dimethylbenzene and embedded in paraffin (Beijing Jiu Zhou Bai Lin Biological Technology Co., Ltd.). The samples were then cut into $4-\mu \mathrm{m}$-thick sections. The sections were stained with hematoxylin and eosin for $10 \mathrm{~min}$ at room temperature $\left(20-25^{\circ} \mathrm{C}\right)$ and images were captured under a microscope (magnification, x100). A lung biopsy revealed numerous calcified bodies in the alveolar spaces (Fig. 1C and D). A ${ }^{99 m}$ Technetium-methylene diphosphonate (Tc-MDP) bone scan revealed increased in take of radiation in the lungs (data not shown). A pulmonary function test (PFT), a SLC34A2 gene test, an oxygen saturation $\left(\mathrm{SO}_{2}\right)$ test and a tuberculin skin test were all normal (data not shown). Routine blood biochemistry tests, including serum calcium and phosphorus concentrations, and hepatic, renal and parathyroid functions were also normal (data not shown). Arterial blood gas (ABG) measurement and echocardiography were not performed due to refused consent by the patient. The patient was born naturally with no asphyxia at birth or dysgnosia during growth. However, the patient's height and weight were notably lower than the national average for Chinese children (10). Based on the typical appearance on HRCT and the calcified bodies identified in the BALF and alveolar spaces, the patient was diagnosed with PMA. However, as there were no serious clinical symptoms at the time of hospital admittance, the patient did not accept any treatment. At present no follow up has occurred.

Case 2. A 31-year-old female from a non-consanguineous family presented to PUMCH in April 2015 due to an abnormal appearance on a chest X-ray observed during a medical examination 1 year prior. The patient did not present with any respiratory symptoms such as dyspnea on exertion or a cough. Physical examination revealed decreased pulmonary sounds in the lower zones and P2>A2 was heard during heart auscultation. The HRCT revealed diffuse, scattered, hyperdense micronodules throughout the whole lungs, accompanied by interlobular septal and bilateral pleural calcific thickening, subpleural cysts between the ribs and calcified parenchyma, referred to as the 'black pleura' sign (Fig. 2A and B). The BAFL examination (performed as above) revealed that the classification of inflammatory cells was normal and the ratio of cluster of differentiation $(\mathrm{CD}) 4^{+} / \mathrm{CD} 8^{+} \mathrm{T}$ cells was 0.9 (reference range, 0.9-2.0), but a calcified body was detected (Fig. 2C). ABG measured pH 7.378, $\mathrm{PaCO}_{2} 37.0 \mathrm{mmHg}, \mathrm{PaO}_{2}$ $88.1 \mathrm{mmHg}, \mathrm{SO}_{2} 96.4 \%$ and $\mathrm{HCO}_{3} 22 \mathrm{mmol} / 1$ of room air (data not shown). Lung tissue pathology and SLC34A2 gene testing were lacking as a positive diagnosis of PMA had been reached based on the tests performed making invasive surgery unnecessary Gene testing was not performed on this patient due to economic constraints. A ${ }^{99 \mathrm{~m}} \mathrm{Tc}-\mathrm{MDP}$ bone scan revealed a diffusely increased intake of radiation in the lungs. An echocardiograph revealed mild enlargement of the right ventricle (anteroposterior diameter, $32 \mathrm{~mm}$ ), with mild pulmonary hypertension (pulmonary systolic pressure, $37 \mathrm{mmHg}$ ) (data not shown). PFT and routine blood biochemistry were normal (data not shown). In the patient's local hospital they were misdiagnosed with pulmonary tuberculosis (TB) due to the appearance of miliary nodules on HRCT imaging. Following a positive diagnosis for PAM no medication was prescribed. At the 3 year follow-up the clinical course of the patient's disease remained stable.

Case 3. A 35-year-old male whose parents were consanguineous presented at PUMCH in November 2014 with complaints of a persistent cough, sputum and occasional chest pain for the last 10 years, and breathlessness for the last 3 weeks. A physical examination revealed bibasilar crackles (velcro rales), cyanosis of the lips and clubbing of the fingers (data not shown). HRCT imaging revealed scattered miliary nodules with calcifications, ground-glass opacities, the 'black pleura' sign and pleural calcific thickening (Fig. 3A and B). BALF examination (performed as above) detected a calcified body and the ratio of $\mathrm{CD}^{+} / \mathrm{CD}^{+} \mathrm{T}$ cells was 0.5 (reference range, $0.9-2.0$ ) (data not shown). A biopsy revealed numerous calcified bodies filling the alveolar spaces of the lungs (Fig. $3 \mathrm{C}$ and D). ABG measured pH 7.409, $\mathrm{PaCO}_{2} 39.0 \mathrm{mmHg}, \mathrm{PaO}_{2} 73.1 \mathrm{mmHg}, \mathrm{SO}_{2}$ $95.2 \%$ and $\mathrm{HCO}_{3} 24.5 \mathrm{mmol} / \mathrm{l}$ of room air (data not shown). A PFT revealed defects in the patient's diffusion capacity and a reduced lung transfer factor for carbon monoxide of $74.5 \%$ of the predicted values (normal, $\geq 80 \%$ predicted value), while ventilatory function was normal (data not shown). Genomic DNA was extracted from the peripheral blood of the patient using a GenElute ${ }^{\mathrm{TM}}$ Blood Genomic DNA kit according to the manufacturer's protocol (Sigma-Aldrich; Merck KGaA, Darmstadt, Germany). Using Primer3 software version 4.0.0 (primer3.ut.ee/), 12 pairs of primers were designed (Table I) to amplify the coding exons of the SLC34A2 gene, as well as the intronic flanking sequences. Polymerase chain reaction was used to amplify the DNA according to a previously described method (11). Direct sequencing was performed by an ABI 3100 Genetic analyzer (Applied Biosystems; Thermo Fisher Scientific, Inc., Waltham, MA, USA). The obtained sequence variants were tested for pathogenicity using Polyphen2 (12) and MutationTaster2 (13). This genetic testing identified an intervening sequence $2^{+} 10 \mathrm{G}>\mathrm{A}$ mutation in intron 2 and a heterozygous c. $910 \mathrm{~A}>\mathrm{T}$ mutation in exon 8 of the SLE34A2 gene (Fig. 3E). Routine blood biochemistry, an echocardiograph and a tuberculin test were all normal (data not shown). The patient intermittently, self-administered dextromethorphan, licorice root and ambroxol at undefined doses and frequencies without a marked effect. At the 2-year follow-up, 

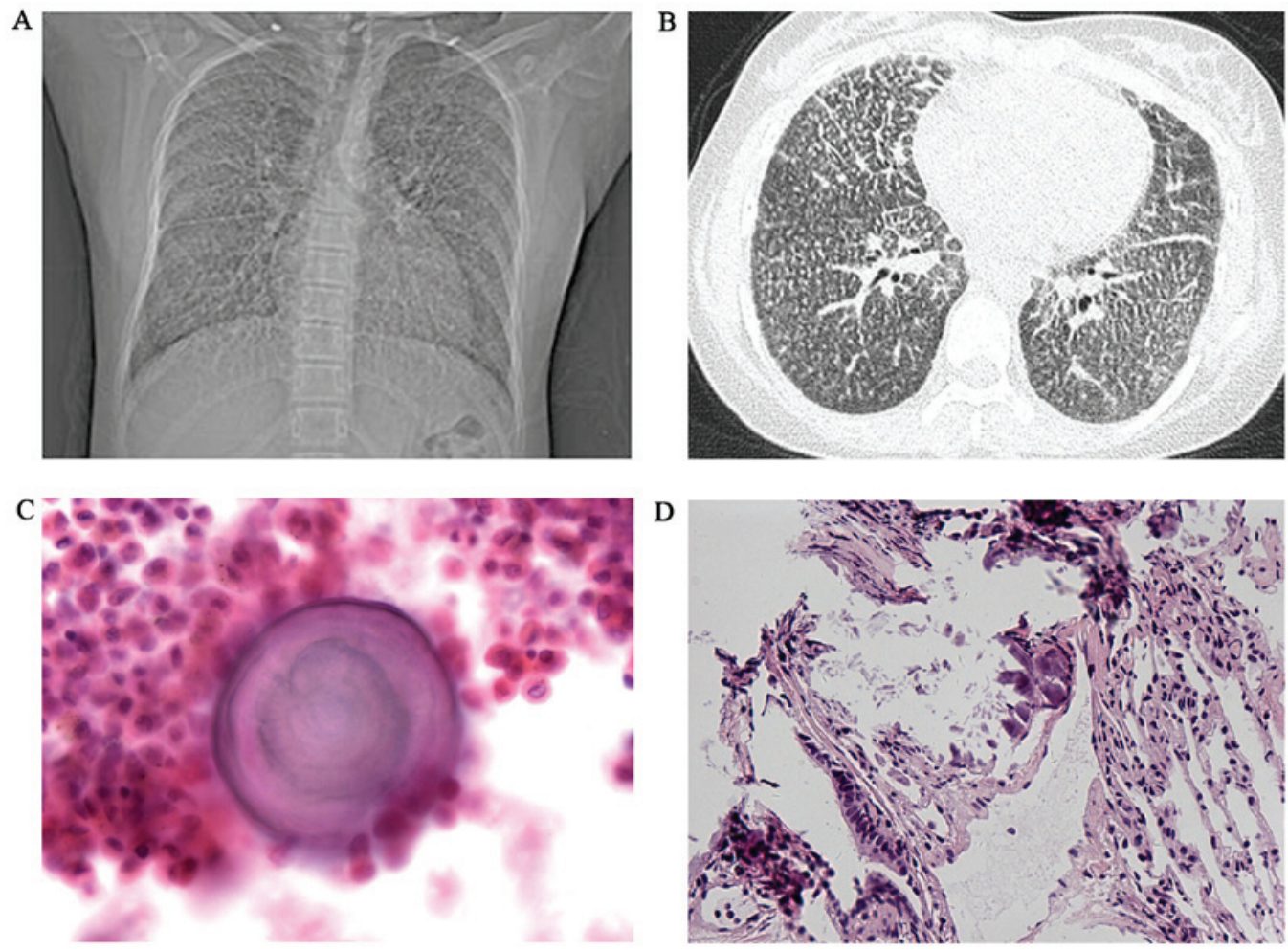

Figure 1. Diagnostic imaging and pathological features of case 1. (A) A chest X-ray exhibiting diffused fine nodules in the lungs, mainly in the middle and lower zones. (B) High resolution computed tomography exhibiting widespread microcalcifications throughout the lungs with ground-glass opacities and septal thickening. (C) Hematoxylin and eosin staining of bronchoalveolar lavage fluid demonstrating a lamellar calcified body with a concentric appearance (magnification, x400). (D) A lung tissue specimen exhibiting numerous introalveolar microliths (magnification, x100).

the patient's dyspnea had advanced with their ABG measuring $\mathrm{PaO}_{2} 69.3 \mathrm{mmHg}$ and $\mathrm{SO}_{2} 94.5 \%$.

\section{Discussion}

PAM is a rare genetic lung disease characterized by widespread sand-like intra-alveolar calcifications (calcospherites) composed of calcium and phosphorus (5), and clinical-radiological dissociation is a hallmark of this disease (14). PAM is present globally and there have been $>1,000$ cases reported to date (3). PAM exhibits no clear sex predilection (2). The majority of patients with PAM have been from Asia, followed by Europe (3). The nation with the highest number of reported cases relative to the population is Turkey, followed by China, Japan, India, Italy and the USA (3). Patients with PAM typically present during the third and fourth decades of life; however, cases have been reported in neonates and octogenarians (14). Familial incidence of PAM has been reported in $35-50 \%$ of cases in Japan, Turkey and Italy (2).

PAM is considered to be a genetic disease due to the inactivating mutations within the SLC34A2 gene identified in patients. This gene is located on chromosome $4 \mathrm{p} 15$ and consists of 13 exons (2). SLC34A2 is primarily expressed in alveolar type II cells (4), but it is also expressed in other epithelial tissues, including mammary glands, the small intestine, kidneys, pancreas, ovaries, liver, testes, placenta and prostate (14). SLC34A2 is the only known sodium-dependent phosphate transporter expressed in the lungs, and it serves a key role in clearing phospholipids from alveolar spaces by transporting phosphate ions into alveolar type II cells (4). The wild-type transporter passages phosphate into the alveolar type II cells in the presence of $\mathrm{Na}$, with a stoichiometry of $3 \mathrm{Na}^{+1}: 1 \mathrm{HPO}_{4}{ }^{-2}$, whereas mutants do not (1). Impaired function or deficiency due to mutations in the SLC34A2 gene leads to a decreased alveolar type II cell uptake of phosphate. This in turn may lead to the formation of intra-alveolar microliths as a result of phosphate-chelating calcium in the extracellular fluid (15). Mutations in the SLC34A2 gene have been detected in almost all tested patients with PAM (2). Mutations have been identified on multiple exons in patients from Turkey, but appear to cluster on exon 8 in cases from China and in exons 7 and 8 in patients from Japan (2). In case 3 of the present study, a heterozygous c.910A $>$ T mutation in exon 8 was detected, which has been reported in previous studies $(11,16)$. PAM has been reported to occur among siblings and cousins in a horizontal pattern, and less frequently between parents and children in a vertical pattern (1). In the cases being reviewed in the present study, the parents of two of the patients were consanguineous.

Upon macroscopic examination, lungs affected by PAM are enlarged, heavy and nonbouyant (2). Sectioning of the lungs often reveals a diffusely calcified, gritty, pleural surface with a studded, fine and granular appearance (2). In the cases reported in the present study, lung biopsies demonstrated characteristic intra-alveolar lamellar microliths, which were extensively composed of $\mathrm{Ca}_{3}\left(\mathrm{PO}_{4}\right)_{2}$; however, small amounts of $\mathrm{CaCo}_{3}, \mathrm{Fe}, \mathrm{Zn}, \mathrm{Al}, \mathrm{SiO}_{2}$ and $\mathrm{Mg}$ are also encountered frequently in PAM microliths (17). Histologically, PAM microliths are periodic acid-Schiff stain positive (18) and consist of calcareous concentric lamellae around a central nucleus with 

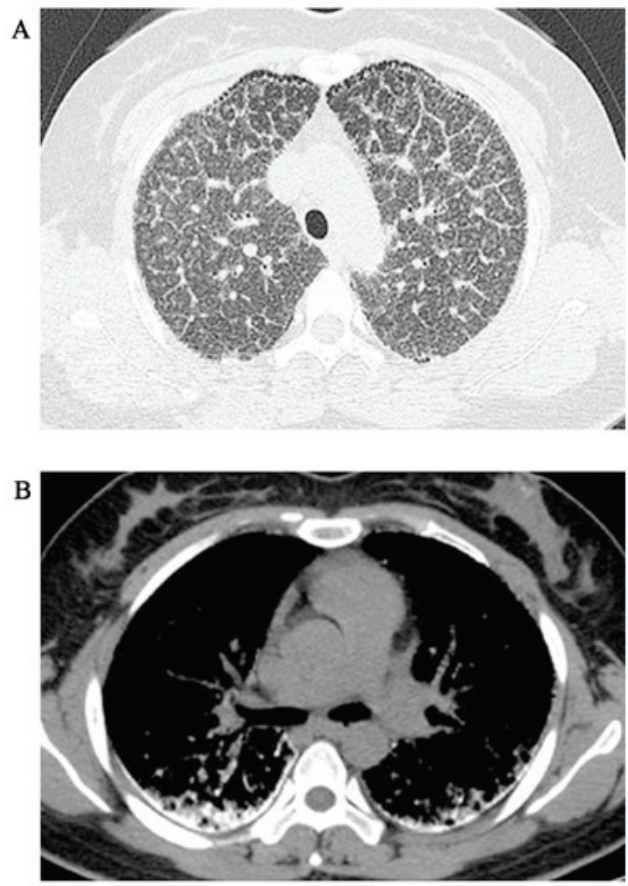

C

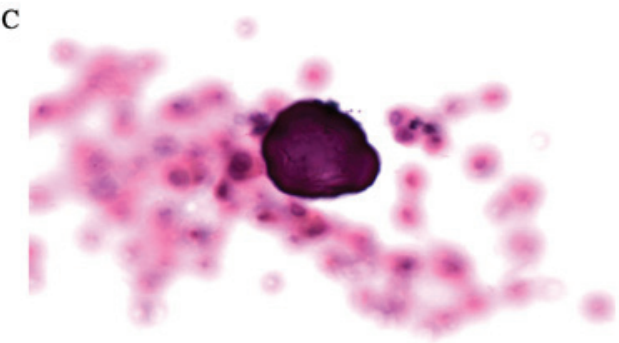

Figure 2. Diagnostic imaging and pathological features of case 2. (A) HRCT imaging of a lung window exhibiting hyperdense micronodular interlobular septal thickening and the black pleura sign in the lungs. (B) HRCT imaging of a mediastinal window demonstrating subpleural intense linear calcification during a bronchoalveolar lavage fluid examination. (C) Hematoxylin and eosin staining of bronchoalveolar lavage fluid demonstrating a lamellar calcified body (magnification, x400). HRCT, high resolution computed tomography.

an amorphous or granular aspect (14). In case 2, the patient did not undergo a lung biopsy; however, the calcified body identified in the BALF was consistent with the pathological diagnosis of PAM. The identification of calcified bodies in BALF may therefore present an alternative method for the histological diagnosis of PAM.

PAM has a relatively indolent although non-modifiable disease progression, and there is often a long period prior to the presentation of any clinical symptoms. As PAM progresses patients commonly develop dyspnea on exertion, other symptoms, including cough, chest pain, hemoptysis, asthenia and pneumothorax, have also been reported. Cyanosis and clubbing of the fingers may be identified in serious cases. A typical clinical manifestation of the disease was observed in case 1 , where the patient only complained of exertional dyspnea. The patient in case 3 , who had been suffering from the disease for a longer period of time and exhibited notable radiographic changes, also suffered from symptoms associated with long-term PAM. The observation of clear cyanosis, clubbing and crackles in this patient indicated the severity of the disease. During the normal progression of PAM microliths may form in early childhood; however, pulmonary function tests are often normal in the early phase. When large volumes of the lung are occupied by microliths it can cause restrictive ventilatory impairment and lead to deterioration of the perfusion capacity over time. This results in hypoxemia, increased arterial $\mathrm{CO}_{2}$ levels (1), pulmonary fibrosis, respiratory failure and ultimately cor pulmonale (19). In case 2, the patient had mild enlargement of the right ventricle and pulmonary hypertension, these symptoms are indicative of a long-term chronic disease and potential failure of the right side of the heart. However, the cases of two patients with PAM reported in a previous study suggest that the disease can be acquired later in life, as their chest radiographs were clinically normal 2 years prior to diagnosis (3). In addition, rapid progression of the disease has also been reported (14).

Routine blood tests, including serum phosphate and calcium, are usually normal in patients with PAM (20). Serum monocyte chemotactic protein-1 (21), surfactant protein (SP)-A and SP-D (22) have been revealed to be elevated in certain patients with PAM. These proteins may potentially serve as diagnostic biomarkers or be used to indicate the activity and progression of PAM.

Extrapulmonary calcifications in the male reproductive system due to PAM can result in testicular atrophy (2), obstructive azoospermia (23) and tumors (24) Calcification of the medullary nephrocalcinosis, nephrolithiasis (25), lumbar sympathetic chain (1), cholelithiasis (26) and cardiac involvements (27) have also been reported in patients with PAM. Microlith deposits outside of the lungs are possibly associated with the penetrance of mutations of the SLC34A2 gene (20). In the present study the three cases did not reveal recognized microlith deposits in other locations.

The typical features of PAM on a plain chest radiographs are a fine, scattered micronodular pattern, producing a 'sandstorm' appearance. Diffuse involvement of both lungs is generally more evident in the middle and lower zones (28). The borders of the heart, diaphragm and vascular tree may be obscured as a result of the dense calcifications (2).

Findings on HRCT can be divided into four stages according to the degree of radiologic severity (29). The first phase, considered as pre-calcific, is without a typical appearance due to the small number and lesser size of the calcifications of the microliths (3). The second phase exhibits the characteristic features of a 'sandstorm' appearance with diffusely scattered calcific micronodules ( $<1 \mathrm{~mm}$ diameter). The microliths tend to distribute throughout the lungs, a greater concentration is usually observed in the medial and inferior regions, although the border of the heart and diaphragm may still be outlined clearly (3). In childhood or adolescent patients these nodules may be larger $(\sim 2-4 \mathrm{~mm})$. The third phase reveals a greater number and volume of opacifications that often lead to the outline of the heart and diaphragm being obscured, as well as interstitial thickening (3). Ground-glass opacity with thickening interlobular septa can sometimes produce the so-called 'crazy-paving' sign (30). The fourth stage is characterized by a notable advance in the number and size of calcific deposits, resulting in intense calcification of the interstitium and 
Table I. Primer sequences for polymerase chain reaction amplification.

\begin{tabular}{lllc}
\hline & \multicolumn{2}{c}{ Primer sequence } & \multicolumn{1}{c}{$\begin{array}{c}\text { Length of amplifie } \\
\text { product (bp) }\end{array}$} \\
\cline { 2 - 3 } Primer name & \multicolumn{1}{c}{ Forward } & \multicolumn{1}{c}{ Reverse } & 477 \\
\hline SLC34A2-1 & CCGTCGGAGCTTTTCTCTCGG & GTCGATCGTAAGAGTGTAGCAGC & 500 \\
SLC34A2-2-3 & GTTGATGCTTTGCAACCAATGG & TGATGACACCCACAGTGAACG & 300 \\
SLC34A2-4 & GCTCATTGCCAAACTTCTCAGG & GCTGGAGAGGGCTTGCTGA & 302 \\
SLC34A2-5 & GGCCTTGGATGGAGACTTCTG & TCCCACCCTCAGATAGACAGG & 270 \\
SLC34A2-6 & GGTAACTTTAGCCTGCCTCCAG & GCATGTCATCTTTGGCTGGTT & 357 \\
SLC34A2-7 & GAGGGTGGCAGATGATACAGG & TGTCAGCTCAGGTAGGGGATG & 243 \\
SLC34A2-8 & CCCTGGGTTTGTGCCTAAATC & CTTCCTTGAAGGCAAGATTAGTT & 241 \\
SLC34A2-9 & CATTGCCTCCCATTCCCCACT & AATAGGTCACCCCCAGACAAC & 300 \\
SLC34A2-10 & TAACAATCTGTAGCCGTGGTGG & GAATCTAAAGGACCCCCACAC & 499 \\
SLC34A2-11-12 & TGTACAACCTCACCCCTAAGCC & AGAGACCAGTTTGCAAGACCATG & 492 \\
SLC34A2-13-1 & TGTGATGCCTGCTAGCTTACCT & CAGCAGCGCATCTGGAAGCAG & 481 \\
SLC34A2-13-2 & CTGCCGAAGAAACTCCAGAACT & CCAAAGGGAATCGAGTTAGGTAG & \\
\hline
\end{tabular}
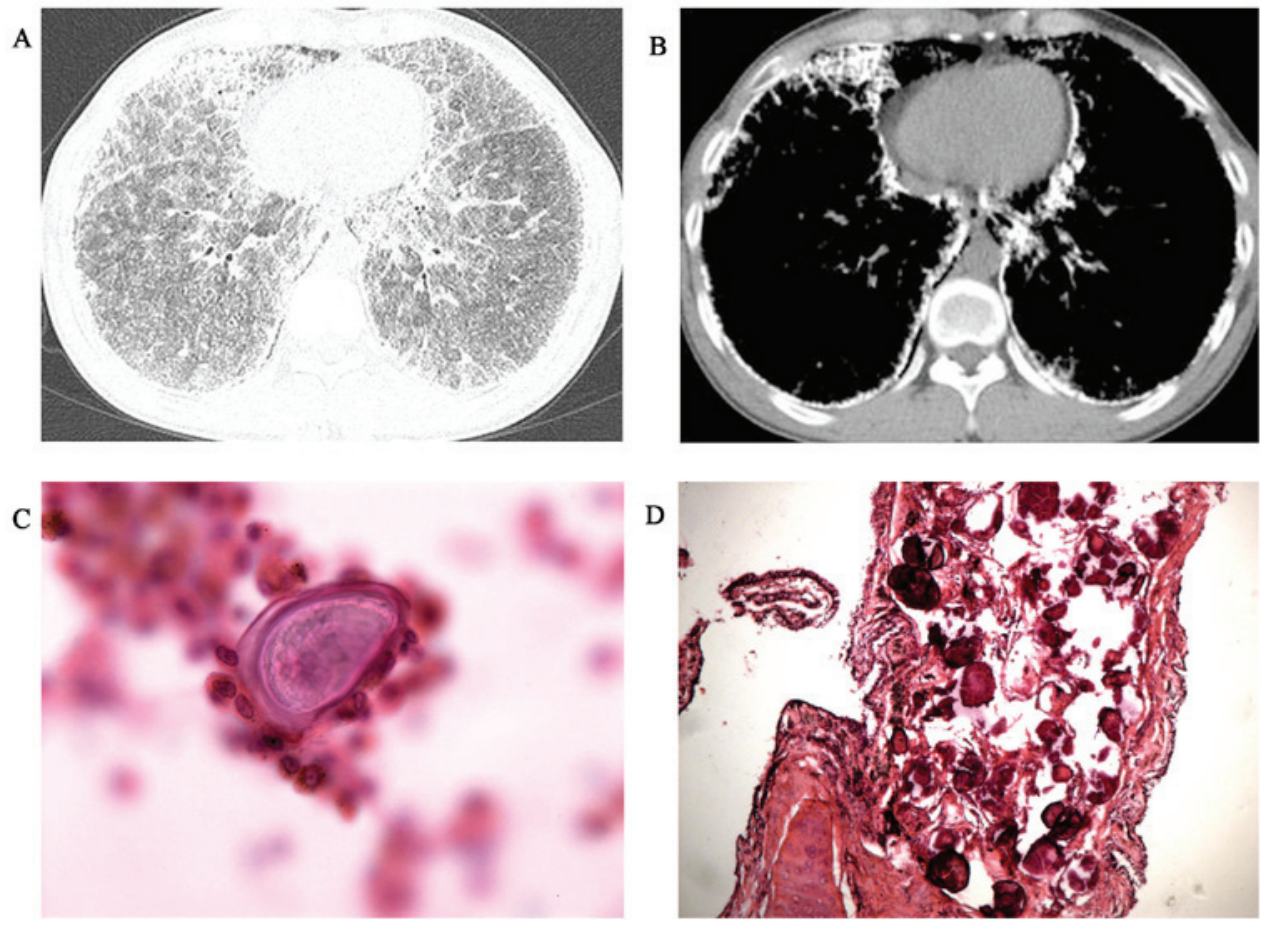

E (1) SLC34A2 intron2, IVS2+10G $>A$, intron mutation

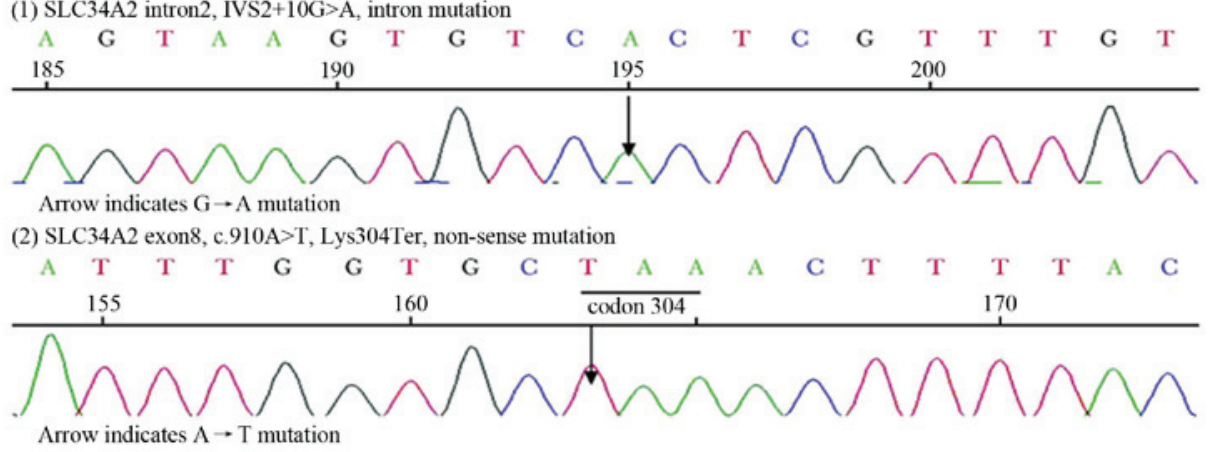

Figure 3. Diagnostic imaging and pathological features of case 3. (A) HRCT imaging of a lung window exhibiting diffuse distribution of small nodules and calcification, mainly in the bilateral lower lobe and subpleural lobe. (B) HRCT imaging of the mediastinal window exhibiting the black pleura sign and calcification deposits along the border of the heart and diaphragm. (C) A hematoxylin and eosin stain of bronchoalveolar lavage fluid exhibiting a calcified body (magnification, x400). (D) A lung specimen exhibiting numerous introalveolar microliths (magnification, x400). (E) Testing of the SLC34A2 gene revealed a mutation IVS2 $2^{+} 10 \mathrm{G}>\mathrm{A}$ in intron 2 and a heterozygous c.910A $>$ T mutation in exon 8 . HRCT, high resolution computed tomography. 
pleural serosa, which gives the overall appearance of "white lungs' (3). The first stage is usually observed in children, the second in adolescence, and the last two stages in the later years of life (3).

A positive diagnosis of PAM can usually be established by observation of a typical sandstorm appearance on chest radiological examinations. A lung biopsy identifying intra-alveolar microliths with a concentric appearance are required in doubtable cases (17). In certain cases microliths can be identified in the BALF or sputum (31), suggesting that this may be a potential alternative to a lung biopsy as the diagnostic procedure for PAM. In case 2, the diagnosis of PAM was established by a combination of radiological observations and BALF, which is consistent with a previous study (32). Testing for a mutation in the SLC34A2 gene may assist when screening family members of the proband patient, but it is not necessary for the diagnosis of PAM.

When dense micronodular and ground-glass opacities are observed in the radiological images, miliary TB, sarcoidosis, pneumoconiosis, pulmonary alveolar proteinosis, pulmonary hemosiderosis and amyloidosis should all be considered as a potential alternative diagnosis to PAM. As in case 2, PAM is often misdiagnosed as TB, particularly in regions where consanguineous marriage is common and TB is highly prevalent (2). Metastatic and dystrophic pulmonary calcifications may be distinguished by a history of chronic renal failure or pathogenic infections (2). The histological appearance of calcareous concentric lamellae around a central nucleus with an amorphous or granular aspect can be also used for distinguishing PAM from metastatic and dystrophic pulmonary calcifications.

To the best of our knowledge, there are currently no guidelines for the treatment of PAM. Systemic steroids, calcium-chelating agents and bronchopulmonary lavage have demonstrated an ineffectiveness at reducing disease progression in previous studies $(3,11)$. Disodium etidronate has previously been administered due to its possible ability to inhibit $\mathrm{Ca}_{3}\left(\mathrm{PO}_{4}\right)_{2}$ precipitation and resolve formed calcifications (8); certain previous studies have demonstrated its ability to improve lung function and radiographic appearances $(8,33)$. However, other reports have revealed that this treatment is not effective $(34,35)$. Lung transplantation can be performed in the end stages of PAM and has previously led to the improvement of right ventricular function (7). The survival rate and recurrence risk following lung transplantation requires additional study; however, to the best of our knowledge, no recurrence of PAM following lung transplantation has been reported to date (3).

The present study described three cases of PAM, a rare genetic disease caused by mutations in the SLC34A2 gene. A brief overview of the disease at different states of progression was also given. PAM reveals a significant clinical-radiological dissociation, meaning that there can be a mild clinical presentation alongside severe radiological changes. Therefore, a diagnosis can be made on the basis of typical radiological features without substantial clinical presentation. The prognosis for individuals diagnosed with PAM is poor due to cor pulmonale and respiratory failure in the later stages of the disease. Lung transplantation currently remains the only effective treatment for this disease.

\section{References}

1. Ferreira Francisco FA, Pereira e Silva JL, Hochhegger B, Zanetti G and Marchiori E: Pulmonary alveolar microlithiasis. State-of-the-art review. Respir Med 107: 1-9, 2013.

2. Saito A and McCormack FX: Pulmonary Alveolar Microlithiasis. Clin Chest Med 37: 441-448, 2016.

3. Castellana G, Castellana G, Gentile M, Castellana R and Resta O: Pulmonary alveolar microlithiasis: Review of the 1022 cases reported worldwide. Eur Respir Rev 24: 607-620, 2015.

4. Izumi H, Kurai J, Kodani M, Watanabe M, Yamamoto A, Nanba E, Adachi K, Igishi T and Shimizu E: A novel SLC34A2 mutation in a patient with pulmonary alveolar microlithiasis. Hum Genome Var 4: 16047, 2017.

5. Mahmood K, Ubaid M and Mahmood A: Pulmonary microlithiasis-A case report. Respir Med Case Rep 19: 112-114, 2016.

6. Qian X, Wu X and Liu X: Pulmonary alveolar microlithiasis with finger clubbing: A case report and literature review. Exp Ther Med 11: 1381-1384, 2016.

7. Samano MN, Waisberg DR, Canzian M, Campos SV, Pêgo-Fernandes PM and Jatene FB: Lung transplantation for pulmonary alveolar microlithiasis: A case report. Clinics (Sao Paulo) 65: 233-236, 2010.

8. Ozcelik U, Yalcin E, Ariyurek M, Ersoz DD, Cinel G, Gulhan B and Kiper N: Long-term results of disodium etidronate treatment in pulmonary alveolar microlithiasis. Pediatr Pulmonol 45: 514-517, 2010.

9. Cakir E, Gedik AH, Özdemir A, Buyukpinarbasili N, Bilgin M and Ozgen IT: Response to disodium etidronate treatment in three siblings with pulmonary alveolar microlithiasis. Respiration 89: 583-586, 2015.

10. Li H, Ji CY, Zong XN and Zhang YQ: Height and weight standardized growth charts for Chinese children and adolescents aged 0 to 18 years. Zhonghua Er Ke Za Zhi 47: 487-492, 2009 (In Chinese).

11. Yin X, Wang H, Wu D, Zhao G, Shao J and Dai Y: SLC34A2 Gene mutation of pulmonary alveolar microlithiasis: Report of four cases and review of literatures. Respir Med 107: 217-222, 2013.

12. Adzhubei IA, Schmidt S, Peshkin L, Ramensky VE, Gerasimova A, Bork P, Kondrashov AS and Sunyaev SR: A method and server for predicting damaging missense mutations. Nat Methods 7: 248-249, 2010.

13. Schwarz JM, Cooper DN, Schuelke M and Seelow D: MutationTaster2: Mutation prediction for the deep-sequencing age. Nat Methods 11: 361-362, 2014.

14. Chu A, Shaharyar S, Chokshi B and Bhardwaj N: Pulmonary alveolar microlithiasis 'stone lungs': A case of clinico-radiological dissociation. Cureus 8: e749, 2016.

15. Field JA, Zhang L, Brun KA, Brooks DP and Edwards RM: Cloning and functional characterization of a sodium-dependent phosphate transporter expressed in human lung and small intestine. Biochem Biophys Res Commun 258: 578-582, 1999.

16. Wang H, Yin X, Wu D and Jiang X: SLC34A2 gene compound heterozygous mutation identification in a patient with pulmonary alveolar microlithiasis and computational 3D protein structure prediction. Meta Gene 2: 557-564, 2014.

17. Mariotta S, Ricci A, Papale M, De Clementi F, Sposato B, Guidi L and Mannino F: Pulmonary alveolar microlithiasis: Report on 576 cases published in the literature. Sarcoidosis Vasc Diffuse Lung Dis 21: 173-181, 2004.

18. Lauta VM: Pulmonary alveolar microlithiasis: An overview of clinical and pathological features together with possible therapies. Respir Med 97: 1081-1085, 2003.

19. Terada T: Pulmonary alveolar microlithiasis with cor pulmonale: An autopsy case demonstrating a marked decrease in pulmonary vascular beds. Respir Med 103: 1768-1771, 2009.

20. Tachibana T, Hagiwara K and Johkoh T: Pulmonary alveolar microlithiasis: Review and management. Curr Opin Pulm Med 15: 486-490, 2009.

21. Saito A, Nikolaidis NM, Amlal H, Uehara Y, Gardner JC, LaSance K, Pitstick LB, Bridges JP, Wikenheiser-Brokamp KA, McGraw DW, et al: Modeling pulmonary alveolar microlithiasis by epithelial deletion of the Npt2b sodium phosphate cotransporter reveals putative biomarkers and strategies for treatment. Sci Transl Med 7: 313ra181, 2015.

22. Takahashi H, Chiba H, Shiratori M, Tachibana T and Abe S: Elevated serum surfactant protein A and D in pulmonary alveolar microlithiasis. Respirology 11: 330-333, 2006. 
23. Kanat F, Teke $\mathrm{T}$ and Imecik O: Pulmonary alveolar microlithiasis with epididymal and periurethral calcifications causing obstructive azospermia. Int J Tuberc Lung Dis 8: 1275, 2004.

24. Kim B, Winter TC III and Ryu JA: Testicular microlithiasis: Clinical significance and review of the literature. Eur Radiol 13 : 2567-2576, 2003.

25. Pant K, Shah A, Mathur RK, Chhabra SK and Jain SK: Pulmonary alveolar microlithiasis with pleural calcification and nephrolithiasis. Chest 98: 245-246, 1990.

26. Piesiak P, Kasibowska-Kuźniar K and Jankowska R: Pulmonary alveolar microlithiasis in a patient with urolithiasis and cholelithiasis. Pneumonol Alergol Pol 69: 285-289, 2001 (In Polish).

27. Jönsson ÅL, Hilberg O, Bendstrup EM, Mogensen S and Simonsen U: SLC34A2 gene mutation may explain comorbidity of pulmonary alveolar microlithiasis and aortic valve sclerosis. Am J Respir Crit Care Med 185: 464, 2012.

28. Chan ED, Morales DV, Welsh CH, McDermott MT and Schwarz MI: Calcium deposition with or without bone formation in the lung. Am J Respir Crit Care Med 165: 1654-1669, 2002.

29. Castellana G, Castellana R, Fanelli C, Lamorgese V and Florio C: Pulmonary alveolar microlithiasis: Clinical and radiological course of three cases according to conventional radiology and HRCT. A hypothesis for radiological classification. Radiol Med 106: 160-168, 2003 (In English, Italian)

30. Gasparetto EL, Tazoniero P, Escuissato DL, Marchiori E, Frare E Silva RL and Sakamoto D: Pulmonary alveolar microlithiasis presenting with crazy-paving pattern on high resolution CT. Br J Radiol 77: 974-976, 2004.
31. Chatterji R, Gaude GS and Patil PV: Pulmonary alveolar microlithiasis: Diagnosed by sputum examination and transbronchial biopsy. Indian J Chest Dis Allied Sci 39: 263-267, 1997.

32. Monabati A, Ghayumi MA and Kumar PV: Familial pulmonary alveolar microlithiasis diagnosed by bronchoalveolar lavage. A case report. Acta Cytol 51: 80-82, 2007.

33. Ozçelik U, Gülsün M, Göçmen A, Ariyürek M, Kiper N, Anadol D and Cobanoğlu N: Treatment and follow-up of pulmonary alveolar microlithiasis with disodium editronate: Radiological demonstration. Pediatr Radiol 32: 380-383, 2002

34. Mariotta S, Guidi L, Mattia P, Torrelli L, Pallone G, Pedicelli G and Bisetti A: Pulmonary microlithiasis. Report of two cases. Respiration 64: 165-169, 1997.

35. Jankovic S, Pavlov N, Ivkosic A, Erceg I, Glavina-Durdov M, Tocilj J, Dragisic-Ivulic S and Primorac D: Pulmonary alveolar microlithiasis in childhood: Clinical and radiological follow-up. Pediatr Pulmonol 34: 384-387, 2002 .

This work is licensed under a Creative Commons Attribution-NonCommercial-NoDerivatives 4.0 International (CC BY-NC-ND 4.0) License. 